\title{
Repensando o Desenvolvimento de Tecnologias Computacionais de Apoio à Aprendizagem ao Longo da Vida
}

\section{Aracele Garcia de Oliveira Fassbinder ${ }^{1,2}$, Maria Lydia Fioravanti ${ }^{1}$, Camila Dias de Oliveira $^{1}$, Maurício M. Arimoto ${ }^{1,3}$, Ellen Francine Barbosa ${ }^{1}$}

${ }^{1}$ Instituto de Ciências Matemáticas e de Computação, Universidade de São Paulo (ICMC/USP)

${ }^{2}$ Instituto Federal de Educação, Ciência e Tecnologia do Sul de Minas Gerais (IFSULDEMINAS - Campus Muzambinho)

${ }^{3}$ Universidade Estadual do Norte do Paraná, Centro de Ciências Tecnológicas (CCT)

aracele.fassbinder@usp.br; mlfioravanti@usp.br; oliveiracamiladias@gmail.com; mauricioarimoto@gmail.com; francine@icmc.usp.br

\begin{abstract}
This paper presents gaps that have hindered the effectiveness of Lifelong Learning, but that can still be minimized with future and deeper research in Computer Science. Are presented new perspectives of research for the next 10 years, specifically in development of educational modules and environments to support teaching and learning.
\end{abstract}

Resumo. Este artigo discute lacunas que têm impedido a efetividade da Aprendizagem ao Longo da Vida, mas que ainda podem ser minimizadas com pesquisas futuras na área de computação. São apresentadas novas perspectivas de investigação para os próximos 10 anos, especificamente nas linhas de desenvolvimento de módulos educacionais e ambientes de apoio ao ensino e aprendizagem.

\section{Contexto}

O atual cenário mundial, o qual tem sido marcado por rápidas mudanças tecnológicas e constante redefinição de normas sociais, econômicas e políticas, têm impactado diversos aspectos associados à aprendizagem. É improvável pensar que a aquisição de conhecimento e desenvolvimento de habilidades aconteçam apenas dentro de ambientes formais, tais como escolas e universidades [Valente 2001]. Portanto, sistemas educacionais e de formação, bem como modelos de aprendizagem, devem se adaptar para responder aos anseios da sociedade do conhecimento.

A ideia de Aprendizagem Continuada ou Aprendizagem ao Longo da Vida (ALV), do inglês lifelong learning, tem sido utilizada, por exemplo, para caracterizar estratégias que promovam nos indivíduos a busca permanente, autônoma e rápida por novos conhecimentos, desenvolvimento de habilidades, competências, valores e atitudes, em qualquer fase de suas vidas [Valente 2001, Alheit and Dausien 2006]. Entretanto, fazer isso acontecer na prática requer das pessoas a aquisição e, posteriormente, a manutenção de competências e habilidades que se inter-relacionam, tais como metacognição, aprendizagem colaborativa, autorregulação da aprendizagem, resolução de problemas, criatividade e motivação intrínseca, dentre outras. Em outras palavras, isso significa aprender a desenvolver uma postura que favoreça um engajamento em adquirir e aplicar conhecimento, maior consciência dos próprios caminhos de estudo e aprendizagem, e demanda 
ambientes computacionais de aprendizagem capazes de apoiá-los neste processo de planejamento e autogerenciamento do percurso de aprendizagem por diversos cursos, níveis e fases de um sistema educacional e da vida pessoal e profissional [Karoudis and Magoulas 2017].

Considerando essa demanda computacional que apoie o desenvolvimento de habilidades e competências úteis para a incorporação da ALV, diversas tecnologias surgiram e vêm se desenvolvendo (plataformas e aplicações multimídia interativas, tecnologias sociais e colaborativas, computação ubíqua, computação em nuvem, cursos e recursos educacionais abertos, sistemas tutores inteligentes, dentre outras). Elas buscam acompanhar os avanços sociais, tecnológicos e se adaptar às necessidades de ensino-aprendizagem de cada indivíduo, com o objetivo de proporcionar maior interação entre os envolvidos, tornando a troca de conhecimento possível e efetiva.

Entretanto, mesmo com todo o investimento em tecnologias computacionais para esse contexto, a aprendizagem incorporada ao longo de todos os momentos da vida dos indivíduos ainda não é uma realidade ampla e efetiva no século XXI [Demirel and Akkoyunly 2017; Kaplan 2017]. O Government Office for Science do Reino Unido ${ }^{1}$, por exemplo, pontua algumas lacunas correntes, tais como pessoas consideradas altamente habilidosas, mas que não conseguem usá-las na prática; também destaca a visão dos empregadores, os quais afirmam o despreparo dos estudantes que acabaram de se formar; a perda da motivação por aprender à medida em que envelhecem, dentre outras. Já sob a perspectiva computacional, Karoudis e Magoulas (2017) destacam que os ambientes computacionais precisam ser desenvolvidos com o objetivo de apoiar inovadores frameworks para a ALV, tais como integração entre trabalho e aprendizagem, aprendizagem autorregulada, motivação, aprendizagem colaborativa e organizacional.

Sendo assim, um grande desafio que a comunidade de Computação aplicada à Educação ainda precisa considerar, para os próximos 10 anos, é repensar a forma como tecnologias computacionais, as quais deveriam apoiar a ALV, têm sido projetadas, desenvolvidas e validadas. Nesse cenário, duas linhas principais serão consideradas: i) a elaboração de módulos educacionais e ii) o projeto e desenvolvimento de ambientes de apoio ao ensino e aprendizagem.

Este trabalho tem como objetivo examinar os desafios dessas duas linhas, sob o ponto de vista da aderência às competências e habilidades inerentes da ALV, as quais estão sempre em evolução. Isso demanda uma reforma e uso inovador de pedagogias e tecnologias emergentes, a fim de fornecer aos indivíduos serviços mais adequados, que os ajudem a melhorar suas experiências de aprendizagem.

O artigo está organizado da seguinte forma: a Seção 2 destaca barreiras atuais e vindouras, que impedem a efetividade da ALV, e que a área de Computação pode continuar ajudando a resolver. Já a Seção 3 discute atuais desafios da área de computação que precisam ser aprofundados. Soluções para esses desafios computacionais são descritos na Seção 4. A Seção 5 apresenta algumas propostas para avaliar as ações. Finalmente, a Seção 6 sintetiza as principais ideias deste trabalho.

\section{Necessidades e Desafios da Aprendizagem ao Longo da Vida}

Diversos agentes ${ }^{2,3}$ da sociedade estão empenhados em criar subsídios para facilitar a ALV, com o

\footnotetext{
1 https://www.gov.uk/government/collections/future-of-skills-and-lifelong-learning

$3 \mathrm{http}: / /$ www.uil.unesco.org/

$3 \mathrm{http} / /$ ec.europa.eu/education/lifelong-learning-programme_pt
} 
intuito de promover mudanças na sociedade, tornando-a mais sustentável, inclusiva, bem como econômica e culturalmente mais desenvolvida. No Brasil, sob o ponto de vista político, grande parte das ações ainda estão voltadas para grupos específicos da sociedade, tais como a Formação Continuada de Professores ${ }^{4}$, repositório de objetos de aprendizagem ${ }^{5}$ e inclusão social ${ }^{6}$.

Já sob o ponto de vista da Pesquisa e Desenvolvimento (P\&D), o quarto grande desafio da Sociedade Brasileira de Computação ${ }^{7}$, definido como "Acesso participativo e universal do cidadão brasileiro ao conhecimento", busca incentivar o desenvolvimento tecnológico e sua aplicação em prol do acesso universal ao conhecimento. Nesse contexto, é preciso considerar que a ALV requer abordagens tecnológicas holísticas, capazes de apoiar indivíduos de diversas culturas e valores, com diferentes necessidades, estilos de aprendizagem, formação acadêmica e experiências profissionais. Isso se torna desafiador por alguns motivos: (i) é preciso compreender as teorias e fundamentos relacionados ao contexto no qual a ALV surgiu e vem se desenvolvendo, a fim de nortear o desenvolvimento de conteúdos e tecnologias digitais adequadas; (ii) tais conteúdos e tecnologias digitais devem ser projetados para facilitar a ALV e apoiar a criação de oportunidades de aprendizagem formais, não-formais e informais para pessoas de qualquer idade e contexto social; (iii) questões éticas, culturais e sociais também precisam ser consideradas, bem como as diferenças e necessidades das diversas áreas e contextos de atuação, tais como educação continuada em saúde, formação de professores, exatas, etc; (iv) são necessárias mudanças de pensamento sobre formas de ensinar, aprender e avaliar a aprendizagem, e isso implica novas formas de apresentar o conteúdo, adaptar estratégias de ensino para contextos virtuais e móveis, diferentes mecanismos para verificar o conhecimento adquirido e as habilidades desenvolvidas com o uso do conteúdo ou tecnologia digital, dentre outras.

\section{Desafios no desenvolvimento de tecnologias computacionais para a ALV}

Nesta seção, é discutido como a área de Computação vem contribuindo para a ALV, destacando as principais lacunas de investigação de duas linhas principais que têm sido exploradas pelo grupo de pesquisa: i) a elaboração de módulos educacionais (Recursos Educacionais Abertos) e ii) o projeto e desenvolvimento de ambientes de apoio ao ensino e à aprendizagem (Cursos Abertos Online e Massivos e Aprendizagem Móvel).

Módulos educacionais podem ser compreendidos como sendo unidades concisas de estudo, compostas por conteúdos teóricos integrados a atividades práticas e avaliações, cuja disponibilização aos aprendizes é apoiada por recursos tecnológicos e computacionais [B 2014]. Um exemplo são os Recursos Educacionais Abertos (REAs), definidos como materiais de ensino, aprendizagem e pesquisa em qualquer suporte ou mídia, sob domínio público ou licenciados de maneira aberta, permitindo que sejam utilizados ou adaptados por terceiros [UNESCO, 2011]. Eles têm buscado padronizar os conteúdos educacionais disponibilizados de maneira livre e aberta por meio da Internet e, por conta disso, têm recebido uma atenção especial nos últimos anos [B 2011].

Apesar dos benefícios que REAs podem agregar à educação, existem alguns desafios que podem dificultar a ALV que valem ser destacados: i) promover a utilização dos REAs para

\footnotetext{
4 http://portal.mec.gov.br/formacao

$5 \mathrm{http} / / /$ portaldoprofessor.mec.gov.br/index.html

$6 \mathrm{http} / / /$ portal.mec.gov.br/secretaria-de-educacao-continuada-alfabetizacao-diversidade-e-inclusao/programas-e-acoes

http://www.sbc.org.br/documentos-da-sbc/category/141-grandes-desafios
} 
ampliação do acesso à instrução em todos os níveis, contemplando a educação formal e informal [UNESCO, 2012]; ii) ampliar a oferta efetiva de REAs, levando-se em consideração não apenas questões técnicas e de gestão, mas também questões culturais, didáticas e pedagógicas [Dinevski, 2008]; iii) produzir materiais de aprendizagem de qualidade, capazes de serem reutilizados e adaptados a diferentes contextos e situações de aprendizagem. Nesse sentido, iniciativas voltadas para apoiar o design e a criação de REAs de qualidade têm sido investigadas [ABB 2015; ABB 2016]. Entretanto, ainda não há um padrão ou norma de qualidade que possa ser usada como referência por parte de instituições, organizações e profissionais envolvidos no desenvolvimento e disponibilização de REAs [Camilleri, Ehlers and Pawlowski 2014; Kawachi 2014].

Já os Ambientes de Apoio ao Ensino e Aprendizagem podem ser caracterizados como a infraestrutura necessária para efetiva disponibilização de módulos educacionais, fornecendo, de forma geral, apoio à estruturação e apresentação dos conteúdos educacionais e à condução de atividades didáticas, de avaliação e de colaboração [B 2014]. Como exemplo, pode-se considerar os Cursos Abertos Online e Massivos $\left(\mathrm{MOOCs}^{8}\right)$ e a infraestrutura relacionada à Aprendizagem Móvel.

MOOCs vêm desempenhando, desde 2012, um importante papel no apoio à aprendizagem ao longo da vida. Eles provêm oportunidades de colaboração e desenvolvimento profissional e acadêmico. O panorama atual das pesquisas sobre MOOCs inclui, por exemplo, o desenvolvimento e oferta de cursos, construção de plataformas específicas (por exemplo Tim Tec ${ }^{9}$, Google Course Builder $^{10}$, Coursera ${ }^{11}$, MiríadaX ${ }^{12}$, etc) e validação qualitativa da aprendizagem [FDB 2014; Fassbinder et al. 2016]. Entretanto, apesar da popularidade dos MOOCs, existem problemas que precisam ser minimizados, tais como o uso de abordagens pedagógicas tradicionais na construção de mapas de aprendizagem; a carência de funcionalidades inovadoras nas plataformas de MOOCs, que permitam novas formas de avaliar os estudantes e estimulem a autorregulação da aprendizagem, criatividade e inovação; as configurações das plataformas atuais e outras limitações para interligar atividades síncronas, práticas e presenciais. Adicionalmente, a acreditação de habilidades e competências adquiridas em contextos não-formais e informais de aprendizagem também é um desafio. Se por um lado existe uma demanda pelo reconhecimento do conhecimento adquirido nesse contexto, existe uma desconfiança sobre a certificação da qualidade e validade de documentos emitidos por instituições não formalmente autorizadas. Embora a resolução dessa questão esbarre em questões burocráticas e em interesses institucionais, a computação pode ajudar ainda mais.

A aprendizagem móvel também emergiu, nos últimos anos, como ferramenta de apoio ao ensino e aprendizagem. Entre os benefícios que ela proporciona, podemos citar: desperta o interesse do estudante, estimula a interação social provendo mecanismos de comunicação entre os usuários da aplicação, instiga discussão sobre os materiais dos cursos, além de proporcionar maior autonomia para organizar sua rotina de estudos [Al-Ani, Hameed and Faisal 2013; Huang, Yen-Ting and ShuChen 2010; Picek and Matija 2013]. Por outro lado, a aprendizagem móvel também possui algumas limitações, que variam de acordo com a sua aplicação e o contexto no qual ela está inserida. Limitações técnicas incluem lacunas no hardware, baixa capacidade de armazenamento, autonomia

\footnotetext{
${ }_{9}^{8}$ Do inglês Massive Open Online Courses.

http://timtec.com.br/pt/funcionalidades/

$11 \mathrm{https}$ ://edu.google.com/openonline/index.html

11 https://www.coursera.org

2 https://miriadax.net/cursos
} 
da bateria, tamanho das telas e dos teclados, cobertura da rede móvel, banda estreita e alto custo de acesso [Al-Ani, Hameed and Faisal 2013; Huang, Yen-Ting and Shu-Chen 2010; Picek and Matija 2013]. Já as limitações não técnicas incluem a dificuldade dos indivíduos em iniciar com a tecnologia dos dispositivos móveis, a falta de motivação de estudar fora do ambiente de ensino formal, bem como uma diversidade de recursos nos dispositivos que podem distrair o usuário facilmente. Algumas iniciativas têm sido investigadas e aplicadas para mitigar estas limitações, tais como o desenvolvimento e validação de arquiteturas de referência e arquiteturas de software que apoiem o projeto e desenvolvimento de ambientes educacionais móveis, bem como ambientes educacionais móveis funcionais desenvolvidos com base nessas arquiteturas [Fioravanti et al. 2017], desenvolvimento e validação de linhas de produto de software que apoiem o projeto e desenvolvimento de aplicações educacionais móveis [Falvo Júnior et al. 2014; MB 2017] e a proposição de frameworks para aprendizagem móvel considerando o contexto de ALV [Nordin, Embi and Yunus 2010].

\section{Ações para minimizar os desafios computacionais apresentados}

Algumas soluções gerais, considerando os desafios apresentados nas Seções 2 e 3, envolvem ações em pelo menos quatro linhas de investigação, a saber: (i) tecnológica, por meio da pesquisa em novos métodos, padrões, técnicas e modelos de desenvolvimento de conteúdo e tecnologias educacionais. Tais tecnologias deveriam considerar a construção de novas funcionalidades que busquem apoiar o desenvolvimento de habilidades metacognitivas e de autorregulação da aprendizagem, bem como aquelas voltadas à avaliação e acompanhamento, que encorajam os estudantes a refletirem sobre como incorporar competências e habilidades no mercado de trabalho e no desenvolvimento da comunidade onde vivem; construção de sistemas interoperáveis, modelagem de perfis de aprendizes que possam servir diversos sistemas e-learning; modelos de arquiteturas de software que facilitem a cooperação e colaboração entre diferentes níveis de aprendizagem (formal, não-formal, informal); e serviços personalizados para ambientes de apoio à aprendizagem continuada; desenvolvimento de Sistemas Inteligentes, uso de dados para predizer e verificar o comportamento dos estudantes [Chauhan 2014]; (ii) educacional e pedagógico, por meio da investigação de modelos teóricos que forneçam subsídios para a definição de aprendizagem continuada e que possam ser transformados em requisitos que apoiem o desenvolvimento de tecnologias digitais educacionais; (iii) formação e capacitação, por meio da inclusão, nos cursos da área de Computação e afins, de disciplinas com enfoque multidisciplinar na construção de tecnologias educacionais, bem como aumento do fomento aos projetos de pesquisa e extensão nesse contexto; (iv) design universal e acessibilidade, por meio da relação das linhas de trabalho anteriormente citadas, a fim de promover o desenvolvimento de materiais e plataformas acessíveis a qualquer tipo de usuário (seja com deficiência ou algum tipo de limitação), seguindo princípios e métodos adequados para facilitar e favorecer a ALV.

Por outro lado, considerando soluções específicas envolvendo REAs, destacam-se as seguintes ações: (i) investigar e desenvolver mecanismos que propiciem maior qualidade e produtividade do processo de desenvolvimento durante a elaboração dos REAs; (ii) desenvolver e fornecer REAs de qualidade para várias áreas e domínios de conhecimento, tais como Engenharia de Software, Engenharia de Computação, Sistemas de Informação, Matemática e Estatística, além de expandir sua utilização para a Educação Básica e Educação Profissional; (iii) investigar e propor 
modelos e abordagens pedagógicas que se adequem à filosofia ALV; (iv) investigar e desenvolver mecanismos de avaliação e garantia de qualidade específicos para REAs; (v) promover a utilização de técnicas das áreas de Engenharia de Software e IHC (Interação Humano-Computador) para avaliação de usabilidade e acessibilidade dos REAs, com foco em diferentes tipos de usuários; (vi) investigar e desenvolver repositórios acessíveis e mecanismos de busca eficientes para facilitar a descoberta e o reúso de REAs; (vii) investigar e propor modelos de negócio sustentáveis para REAs; (viii) fomentar a inserção da cultura digital e das TICs nas escolas e universidades; e (ix) promover a adoção de software livre e conteúdos abertos por meio de cursos e palestras.

Adicionalmente, no contexto de MOOCs, (i) desenvolver e validar frameworks de apoio à construção de mapas de aprendizagem pautados em uma visão holística e integrada dos principais elementos ligados às metodologias ativas de ensino aprendizagem, colaboração, autorregulagem da aprendizagem, metacognição, avaliação contextualizada, criatividade e inovação; (ii) desenvolver ou adaptar plataformas de MOOCs que incluam recursos que permitam novas formas de avaliação dos estudantes, bem como suportem diferentes estratégias pedagógicas; (iii) desenvolver novas arquiteturas de plataformas que possibilitem a personalização de caminhos de aprendizagem continuada, integrando experiências formais, não-formais e informais, tal como discutido em [Karoudis and Magoulas 2017]. Tais arquiteturas devem agregar fundamentos validados de modelos pedagógicos já existentes e prover facilidade de inclusão de novas ideias a partir de pedagogias emergentes.

Já no âmbito da aprendizagem móvel, (i) propor padrões e/ou linguagens de padrões que forneçam subsídios para a definição de requisitos pedagógicos tanto de ambientes quanto de aplicações móveis educacionais; (ii) criar diretrizes para a produção de conteúdo para aplicações educacionais móveis; (iii) investigar questões de acessibilidade, usabilidade e segurança nas aplicações educacionais móveis; (iv) estímulo a docentes e tutores para utilizar a aprendizagem móvel como ferramenta de apoio.

\section{Avaliação das Ações e Resultados}

Definir formas de avaliar intervenções nesse contexto poderia ser considerado um desafio a ser investigado, devido à complexidade e dificuldade em definir quantitativamente os impactos das mudanças na sociedade como um todo. Ainda assim, a fim de avaliar os resultados das ações de pesquisa e desenvolvimento para resolver, ou ao menos minimizar, o desafio proposto neste trabalho, sugere-se: (i) monitorar e avaliar o impacto do uso de tecnologia no apoio à ALV sob o ponto de vista do desenvolvimento social, cultural e econômico das sociedades relacionadas; (ii) definir critérios que facilitem a avaliação desse impacto sobre o nível e qualidade do conhecimento adquirido, bem como habilidades desenvolvidas pelo aprendiz; e (iii) analisar os incentivos e políticas públicas para o fomento de projetos nesse contexto. Adicionalmente, dados quantitativos podem ser utilizados, tais como análise da produção científica da área, eventos específicos, livros e softwares.

\section{Considerações Finais e Perspectivas Futuras}

O uso de tecnologias computacionais como apoio à incorporação da aprendizagem de modo intrínseco e indissociável da vida pessoal e profissional de um indivíduo, em qualquer idade, local 
ou situação, têm sido objeto de pesquisas acadêmicas e projetos sócio-políticos ao redor do mundo, há mais de duas décadas. Entretanto, considerando a complexidade dessa abordagem, o seu impacto para a transformação de uma sociedade, as mudanças tecnológicas, sociais e diversos fatores que afetam sua efetividade, podemos pontuar que ações de investigação nesse contexto estão longe de serem total e amplamente exploradas. Este artigo buscou fomentar esse debate por meio da apresentação de algumas lacunas atuais da ALV que ainda podem ser minimizadas com o uso da computação. Foram elencados, também, os desafios correntes da computação, especificamente nas linhas de elaboração de módulos educacionais e desenvolvimento de ambientes de apoio ao ensino e aprendizagem. Reflexões sobre possíveis soluções para tais problemas e formas de avaliar seu impacto também foram destacadas.

\section{Agradecimentos.}

Os autores agradecem o suporte financeiro das agências brasileiras de fomento (FAPESP, FAPEMIG, CAPES e CNPq) e do Instituto Federal de Educação, Ciência e Tecnologia do Sul de Minas Gerais - IFSULDEMINAS.

\section{Referências}

Al-Ani, M. F.; Hameed, S. M. and Faisal, L. (2013) "Students' perspectives in adopting mobile learning at university of bahrain." e-Learning" Best Practices in Management, Design and Development of e-Courses: Standards of Excellence and Creativity".

Alheit, P. and Dausien, B. (2006) Processo de formação e aprendizagens ao longo da vida. Educação e Pesquisa, v. 32, n. 1.

Arimoto, M. M.; Barroca, L. and Barbosa, E. F. (2016) "AM-OER: An Agile Method for the Development of Open Educational Resources", Informatics in Education, v. 15, n. 2 p. 205-233.

Arimoto, M. M.; Barroca, L. and Barbosa, E. F. (2015) "An agile learning design method for open educational resources”, In: 2015 IEEE Frontiers in Education Conference (FIE).

Barbosa, E. F.; Maldonado, J. C. (2011) "Collaborative development of educational modules: A need for lifelong learning", E-Infrastructures and Technologies for Lifelong Learning: Next Generation Environments-G. D. Magoulas. (Org.), p. 37.

Barbosa, E. F. (2014) "Módulos Educacionais e Ambientes de Ensino e Aprendizagem: Contribuições e Perspectivas", Texto de Livre Docência, USP, São Carlos.

Camilleri, A. F.; Ehlers, U. D. and Pawlowski, L. (2014) "State of the art review of quality issues related to open educational resources", Technical report.

Chauhan, A. (2014) "Massive open online courses: Emerging trends in assessment and accreditation", Digital Education Review, n. 25.

Demirel, M. and Akkoyunly, B. (2017) "Prospective teachers lifelong learning tendencies and information literacy self-efficacy”, Educational Research and Reviews, v. 12, p. 329-337.

Dinevski, D. (2008) "Open educational resources and lifelong learning”, 30th International Conference on Information Technology Interfaces, Croatia, pp. 117 - 122. 
Fassbinder, A.G.O.; Delamaro, M. E. and Barbosa, E. F. (2014) "Construção e Uso de MOOCs: Uma Revisão Sistemática", In: Brazilian Symposium on Computers in Education (Simpósio Brasileiro de Informática na Educação-SBIE).

Fassbinder, A. G. O. et al. (2016) "Towards a MOOC Design Model based on Flipped Learning and Patterns: A Case on Introductory Courses", In: XXI Congresso Internacional de Tecnologia Educativa (TISE), Santiago, Chile.

Fioravanti, M. L. et al. (2017) "Towards a Mobile Learning Environment using Reference Architectures." 50th Hawaii International Conference on System Sciences.

Huang, Y.; Yen-Ting L. and Shu-Chen, C. (2010) "Effectiveness of a mobile plant learning system in a science curriculum in Taiwanese elementary education." Computers \& Education.

Falvo Júnior, V. et al. (2014) "Towards the Establishment of a Software Product Line for Mobile Learning Applications." SEKE.

Kaplan, A. (2017) "Lifelong learning: Conclusions from a Literature Review”, International Online Journal of Primary Education (IOJPE).

Kawachi, P. (2014) "Quality Assurance Guidelines for Open Educational Resources: TIPS Framework".

Karoudis, K. and Magoulas, G. D. (2017) "An architecture for smart lifelong learning design”, In: Innovations in Smart Learning. Springer Singapore, p. 109-115.

Nordin, N.; Embi, M. A. and Yunus, M. M. (2010) "Mobile Learning Framework for Lifelong Learning”, Procedia - Social and Behavioral Sciences, Volume 7, 2010, Pages 130-138.

Picek, R. and Matija G. (2013) "Evaluation of the Potential Use of m-learning in Higher Education." Proceedings of the ITI 2013 35th International Conference.

Marcolino, A. S. and Barbosa, E. F. (2017) "Towards a Software Product Line Architecture to Build M-learning Applications for the Teaching of Programming." Proceedings of the 50th Hawaii International Conference on System Sciences.

Unesco (2011) “Guidelines for Open Educational Resources (OERs) in Higher Education”. United Nations Educational, Scientific and Cultural Organization.

Unesco (2012) "Declaração REA de Paris". United Nations Educational, Scientific and Cultural Organization.

Valente, J. A. (2001) "Aprendizagem continuada ao longo da vida: o exemplo da terceira idade", São Paulo: Cortez, p. 27-44. 